\title{
Evaluation of Renal Tissue Oxygenation Using Blood Oxygen Level-Dependent Magnetic Resonance Imaging in Chronic Kidney Disease
}

\author{
Fen Chen ${ }^{a, b}$ Han Yann ${ }^{a, b}$ Fan Yang ${ }^{a, b}$ Li Cheng $^{c}$ Siwei Zhang ${ }^{c}$ Shulin $\mathrm{Li}^{\mathrm{a}}$ \\ Caixia Liu $^{a}$ Kai Xuc Dong Sun ${ }^{\mathrm{a}, \mathrm{b}}$
}

${ }^{a}$ Department of Nephrology, Affiliated Hospital of Xuzhou Medical University, Xuzhou, China; ${ }^{b}$ Department of Internal Medicine and Diagnostics, Xuzhou Medical University, Xuzhou, China; 'Department of Radiology, Affiliated Hospital of Xuzhou Medical University, Xuzhou, China

\section{Keywords}

Blood oxygen level-dependent - Magnetic resonance imaging · Chronic kidney disease $\cdot$ Hypoxia $\cdot$ Methods

\begin{abstract}
Background: Blood oxygen level-dependent magnetic resonance imaging (BOLD-MRI) has been widely used to assess renal oxygenation changes in different kidney diseases in recent years. This study was designed to evaluate and compare renal tissue oxygenation using 2 BOLD-MRI analysis methods, namely, the regional and whole-kidney region of interest (ROI) selection methods. Methods: The study ended up with 10 healthy controls and 40 chronic kidney disease (CKD) patients without dialysis. Their renal BOLD-MRI data were analyzed using whole-kidney $\mathrm{ROI}$ selection method and compared with regional ROI selection method. Results: We found the cortical, medullary, and whole-kidney R2* values were significantly higher in CKD patients than those in controls. Compared with the regional ROI selection method, the whole-kidney ROI selection method yielded higher cortical R2* values in both controls and CKD patients. The wholekidney R2* values of deteriorating renal function group were significantly higher than those in stable renal function group. Conclusions: Cortical and medullary oxygenation was decreased significantly in CKD patients compared with the

healthy controls, particularly in the medulla. The whole-kid-
\end{abstract}

karger@karger.com

www.karger.com/kbr

Karger $\stackrel{\text { ' }}{=}$

BOPEN ACCESS
(C) 2021 The Author(s)

Published by S. Karger AG, Basel

This is an Open Access article licensed under the Creative Commons Attribution-NonCommercial-4.0 International License (CC BY-NC) (http://www.karger.com/Services/OpenAccessLicense), applicable to the online version of the article only. Usage and distribution for commercial purposes requires written permission. ney R2* values were positively correlated with kidney function and inversely correlated with the estimated glomerular filtration rate and effective renal plasma flow. Whole-Kidney R2* value might effectively predict the progression of renal function in patients with CKD.

(C) 2021 The Author(s)

Published by S. Karger AG, Basel

\section{Introduction}

The kidney is a highly perfused organ, and the blood flow in the kidney is mainly distributed in the cortex. The medulla has significantly lower oxygenation because of smaller blood supply and greater oxygen consumption. Constant oxygen consumption, microvascular injury, and inflammation in the renal parenchyma make the kidney vulnerable to low perfusion and hypoxic damage, resulting in renal interstitial fibrosis and aggravating the progression of kidney disease $[1,2]$. Therefore, monitoring of renal oxygenation is helpful for early treatment of kidney disease $[3,4]$.

In the 1990s, blood oxygen level-dependent magnetic resonance imaging (BOLD-MRI) came into being as a

Fen Chen, Han Yan and Fan Yang contributed equally to this work. Kai Xu and Dong Sun contributed equally to this work.
Correspondence to:

Kai Xu, xukaixz@163.com

Dong Sun, sundong126@yahoo.com 
Fig. 1. Forty-two nondialysis patients with CKD were enrolled in the study, the BOLD images of 1 patient were lost and the BOLD images of 1 patient did not conform to the analysis standard. ECT was performed in 29 CKD patients to evaluate their ERPF. Ten healthy volunteers were enrolled in the study. Of the 40 CKD patients included, 29 patients had reexamination of renal function during 6 months to 1 year. The eGFR increased or remained unchanged in 15 patients and decreased in 14 patients. eGFR, estimated glomerular filtration rate; ERPF, effective renal plasma flow; ECT, emission computed tomography.

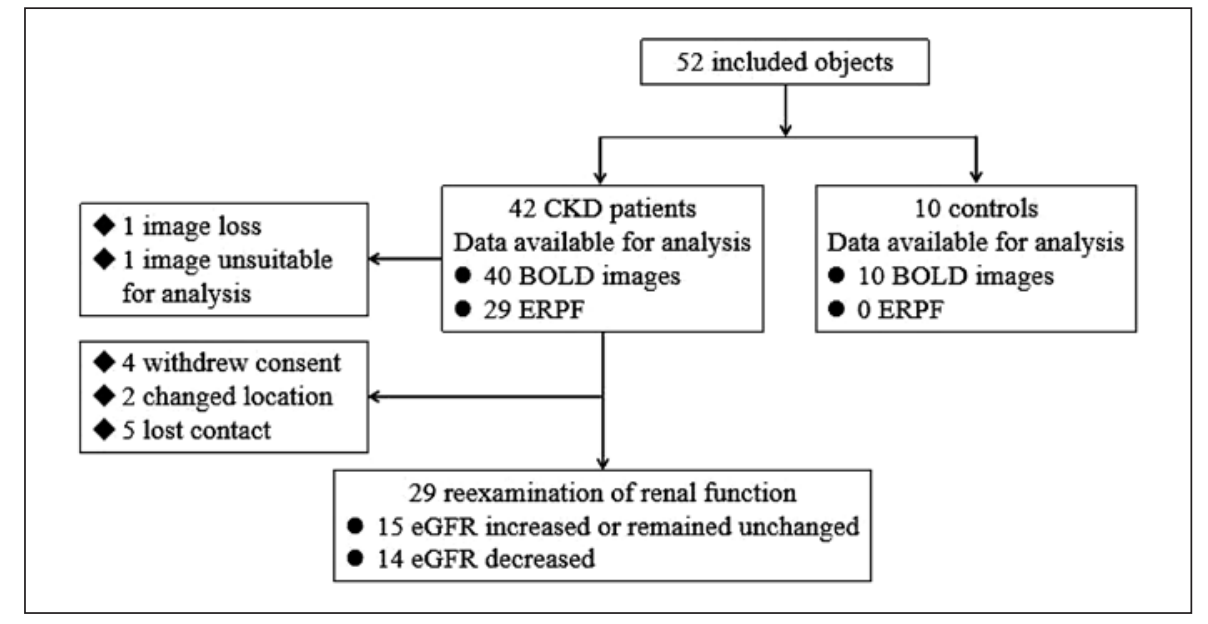

new technology for the noninvasive evaluation of tissue oxygenation [5]. BOLD-MRI is based on paramagnetic deoxyhemoglobin. Deoxyhemoglobin is an endogenous contrast agent that can affect the signal of T2*-weighted MRI scans. As the reciprocal of T2*, R2* is closely related to the content of deoxyhemoglobin in tissues $\left(\mathrm{R} 2^{*}=1 /\right.$ $\mathrm{T} 2 *$ ) [6]. An increase in the R2* value indicates a decrease in the partial oxygen pressure, suggesting tissue hypoxia.

To date, BOLD-MRI has been widely used to assess renal oxygenation changes in different kidney diseases, including ischemic kidney disease [7], hypertensive nephropathy [8], diabetic nephropathy [9], chronic kidney disease (CKD) [10], acute kidney injury [11], and renal allograft rejection [12]. However, there are still no standardized BOLD image analysis methods. For decades, researchers have been exploring accurate and reproducible analysis methods for BOLD-MRI, including regional region of interest (ROI) selection [13], compartmental [14], fractional tissue hypoxia [15], concentric objects [16], and twelve-layer concentric objects $[17,18]$ methods. The regional ROI selection method is a traditional BOLDMRI analysis method. In this method, an ROI is placed in the upper, middle, and lower poles of the cortical and medullary regions, and then the oxygenation is evaluated by obtaining the mean $\mathrm{R} 2 *$ values. As the ROIs are drawn manually, the shape and size of these ROIs vary and could contain cortico-medullary overlap zones, which may affect the accuracy and reproducibility of the R2* values. Saad et al. [19] proposed a new analysis method, called fractional tissue hypoxia, to evaluate the oxygenation changes in patients with atherosclerotic renal artery stenosis. In this method, oxygenation is evaluated by measuring the percentage of R2* values above $30 \mathrm{~s}^{-1}$ in the cortex and renal parenchyma $[7,19,20]$. However, the threshold of R2* values above $30 \mathrm{~s}^{-1}$ is arbitrary, and this threshold may not be applicable to other kidney diseases.

In this study, 2 BOLD-MRI analysis methods, called the regional and whole-kidney ROI selection methods, were used to evaluate renal tissue oxygenation in healthy persons and CKD patients. The correlations between the R2* values and renal function, estimated glomerular filtration rate (eGFR), and effective renal plasma flow (ERPF) in CKD patients were studied to compare the advantages and disadvantages of the 2 methods.

\section{Methods}

\section{Subjects}

The study has been approved by the ethics review board and registered on the Clinical Trials.gov (NCT03321942). All healthy persons and CKD patients signed informed consent. Ten healthy volunteers at the Medical Examination Center of the Affiliated Hospital of Xuzhou Medical University were recruited from December 2015 to December 2017 as controls. At the same time, 42 nondialysis patients with CKD in the Department of Nephrology of the Affiliated Hospital of Xuzhou Medical University were enrolled in the CKD group. Among the CKD patients included in this study, the protopathy was chronic glomerulonephritis for 41 patients and hypertensive nephropathy for 1 patient. Most of the patients were in stage $1-3$ CKD $(n=27)$. The patients maintained their treatment and antihypertensive drugs.

During the first week of this study, we measured the height, weight, and blood pressure of the subjects. The serum glucose, hemoglobin, serum creatinine (Scr), blood urea nitrogen, uric acid, and cystatin $\mathrm{C}$ levels were measured by routine blood and kidney function tests. The kidney volume in CKD patients was estimated using measurements of MRI. The eGFR was calculated by CKD epidemiology collaboration formula. Emission computed tomography was performed in $29 \mathrm{CKD}$ patients to evaluate their ERPF. In the second week of this study, all the participants who had completed the above examinations in the previous week were made 


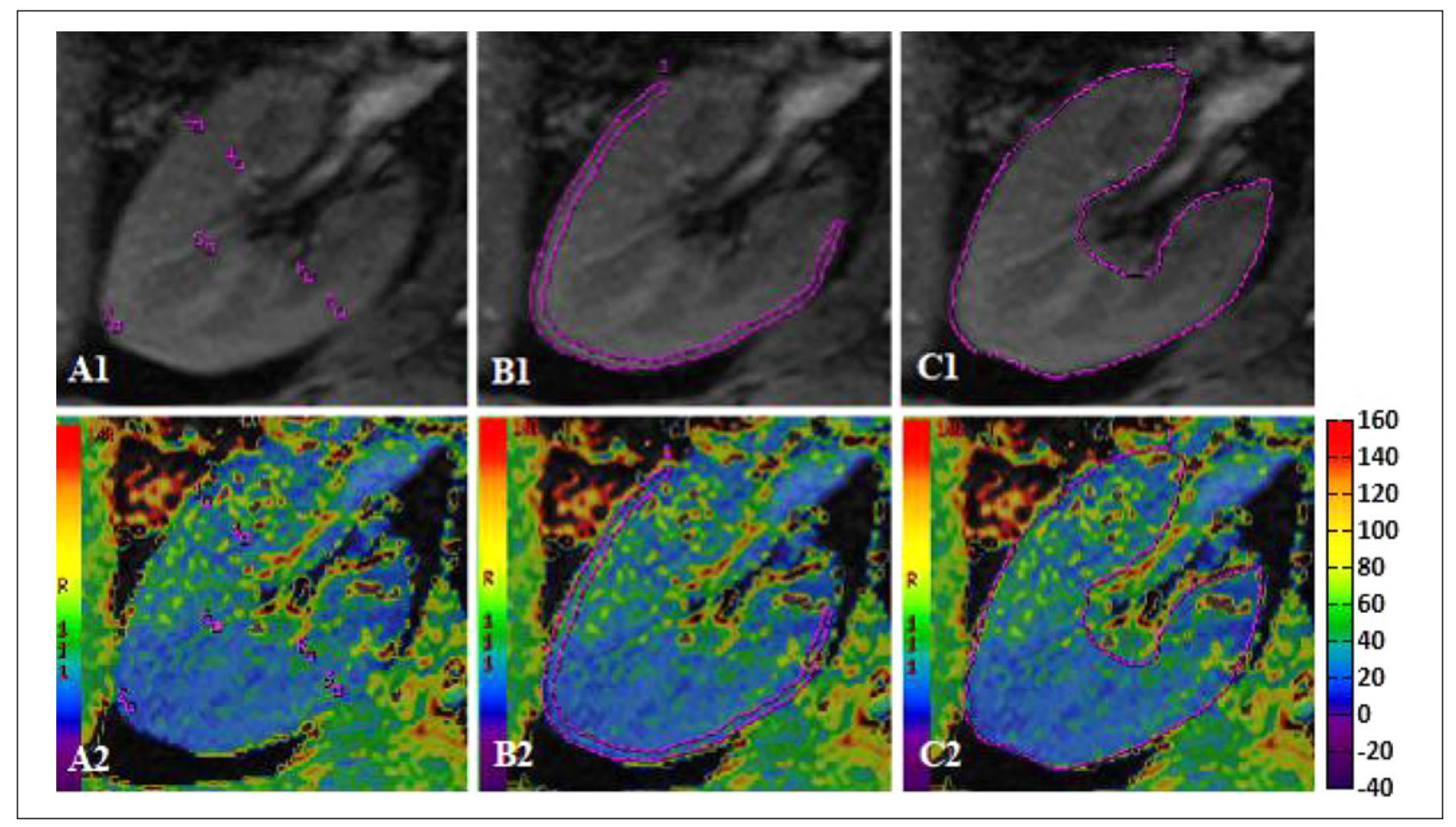

Fig. 2. Regional ROI selection method: A the mean cortical and medullary R2* values are obtained by placing ROIs in the cortex (ROIs 1-3) and the medulla (ROIs 4-6). Whole-kidney ROI selection method: B the cortical $\mathrm{R} 2 *$ value is obtained by placing an ROI containing the wide cortical area. C The whole-kidney R2* value is obtained by placing an ROI over the renal parenchyma (excluding the collection system, cysts, and hilar vessels). ROI, region of interest.

complete BOLD-MRI examination in 1 day. On the day of examination, all participants ate the same food; CKD patients continued previous treatments, including angiotensin-converting enzyme inhibitor or angiotensin-receptor blocker drugs.

In the CKD group, the BOLD images of 1 patient were lost, and the BOLD images of 1 patient did not conform to the analysis standard because of severe atrophy of the kidneys. Finally, $40 \mathrm{CKD}$ patients were included in the analysis. Of the $40 \mathrm{CKD}$ patients included, 29 patients had reexamination of renal function after 6 months to 1 year. The eGFR increased or remained unchanged in 15 patients and decreased in 14 patients. They were divided into stable renal function group and deteriorating renal function group based on eGFR (Fig. 1).

\section{BOLD-MRI Parameters}

During the second week of this study, the controls and CKD patients underwent BOLD-MRI with a 3.0-T system (GE Medical Systems, Discovery MR 750w). BOLD images of coronal slices were acquired through the renal hilum during breath-hold using a multiple gradient echo sequence. The BOLD-MRI parameters were as follows: echoes, 10; repetition time, $47.8 \mathrm{~ms}$; echo time, $1.9 \sim 37.1 \mathrm{~ms}$; flip angle, $20^{\circ}$; slice thickness, $5 \mathrm{~mm}$; interval, $0 \mathrm{~mm}$; field of view, $40 \times 32 \mathrm{~cm}^{2}$; matrix, $96 \times 180$; and number of excitations, 2 . The system automatically generated an R2* pseudocolor map showing a gradual transition from blue to green, yellow, and red from the cortex to the medulla. Blue represents high renal tissue oxygenation, and red represents significant hypoxia, with changes from blue to green, yellow, and red indicating a gradual increase in renal hypoxia.

\section{BOLD-MRI Analysis Methods}

A T2*-weighted image with the clearest demarcation of the cortex and the medulla was selected from among one of the 10 coronal slices. Two methods were used to analyze BOLD images in this study.

\section{Regional ROI Selection Method}

An ROI was placed in the upper, middle, and lower poles of the cortex to measure the $\mathrm{R} 2 *$ values of these areas, and the average of the 3 R2* values was taken as the cortical R2* value. The medullary $\mathrm{R} 2 *$ value was obtained by the same method (Fig. 2 : A1, 2). We attempted to produce ROIs as similar as possible in shape and size.

\section{Whole-Kidney ROI Selection Method}

The R2* values of the cortex were measured with $1 \mathrm{ROI}$ including most cortical areas (Fig. 2: B1, 2). One ROI was placed by tracing the whole renal parenchyma (except the collection system, cysts, and hilar vessels) to measure the whole-kidney R2* value (Fig. 2: C1, 2). 
Table 1. Demographics and clinical characteristics of controls and CKD patients

\begin{tabular}{|c|c|c|c|}
\hline Characteristic & Controls $(n=10)$ & $\mathrm{CKD}(n=40)$ & $p$ value \\
\hline Age, years & $43.2 \pm 14.4$ & $44.2 \pm 14.9$ & 0.85 \\
\hline Age range, years & $24-65$ & $17-74$ & \\
\hline Sex (males/females) & $5 / 5$ & $24 / 16$ & 0.57 \\
\hline Body mass index, $\mathrm{kg} / \mathrm{m}^{2}$ & $24.8 \pm 3.8$ & $24.4 \pm 3.5$ & 0.78 \\
\hline Hypertension & 0 & 33 & \\
\hline \multicolumn{4}{|l|}{ Blood pressure, mm Hg } \\
\hline Systolic blood pressure & $123.6 \pm 6.5$ & $142.3 \pm 21.0$ & $<0.001$ \\
\hline Diastolic blood pressure & $74.8 \pm 6.5$ & $86.8 \pm 11.7$ & $<0.001$ \\
\hline ACEIs or ARBs & 0 & 13 & \\
\hline Alpha blockers & 0 & 9 & \\
\hline Beta blockers & 0 & 16 & \\
\hline Calcium channel blockers & 0 & 29 & \\
\hline Loop diuretics & 0 & 9 & \\
\hline Statins & 0 & 4 & \\
\hline Serum glucose, $\mathrm{mmol} / \mathrm{L}$ & $5.0 \pm 0.48$ & $4.8 \pm 0.47$ & 0.51 \\
\hline Hemoglobin, g/L & $143.8 \pm 19.2$ & $114.6 \pm 26.9$ & 0.002 \\
\hline $\mathrm{Scr}, \mu \mathrm{mol} / \mathrm{L}$ & $62.3 \pm 12.9$ & $255.6 \pm 194.4$ & $<0.001$ \\
\hline Blood urea nitrogen, $\mathrm{mmol} / \mathrm{L}$ & $5.2 \pm 1.6$ & $13.7 \pm 7.8$ & $<0.001$ \\
\hline Uric acid, $\mu \mathrm{mol} / \mathrm{L}$ & $288.1 \pm 95.8$ & $440.3 \pm 132.3$ & 0.001 \\
\hline Cystatin C, mg/L & $0.7 \pm 0.1$ & $1.9 \pm 0.8$ & $<0.001$ \\
\hline $\mathrm{eGFR}, \mathrm{mL} / \mathrm{min}$ & $110.86 \pm 12.78$ & $42.44 \pm 32.86$ & $<0.001$ \\
\hline
\end{tabular}

The data are expressed as the mean \pm standard deviation or as a numerical value. ACEI, angiotensin-converting enzyme inhibitor; ARB, angiotensin-receptor blocker; eGFR, estimated glomerular filtration rate; Scr, serum creatine. The systolic pressure, diastolic pressure, serum creatinine, blood urea nitrogen, uric acid and cystatin $\mathrm{C}$ levels in CKD patients were higher than in controls ( $p<0.001$ vs. control group for all), while the hemoglobin level and eGFR in CKD patients were significantly lower than in controls $(p=0.002$ and $<0.001$, respectively).

Statistical Analysis

SPSS 22.0 statistical software was used to analyze the data, and the results are expressed as the mean \pm standard deviation $(\bar{x} \pm s)$. Independent sample $t$ tests were used to compare age, body mass index, blood pressure, serum glucose, hemoglobin, Scr, blood urea nitrogen, uric acid, cystatin C, and eGFR between the controls and CKD patients. The kidney volume and ERPF of the left and right kidneys in CKD patients were also compared using independent sample $t$ tests. The $\chi^{2}$ test was used for comparing sex between the 2 groups. The R2* values of the left and right kidneys in the controls and CKD patients, evaluated by the 2 BOLD-MRI analysis methods, were compared by variance analysis. The correlations between the whole-kidney R2* value and age, blood pressure, hemoglobin, Scr, blood urea nitrogen, uric acid, cystatin C, eGFR, kidney volume, and ERPF in CKD patients were analyzed by Pearson correlation analysis. $p \leq 0.05$ indicates a statistically significant difference.

\section{Results}

Comparison of the Demographics and Clinical Characteristics of the Controls and CKD Patients

The age, sex, and body mass index of the 2 groups were not significantly different $(p=0.85,0.57$, and 0.78 , respec- tively). The systolic pressure, diastolic pressure, Scr, blood urea nitrogen, uric acid, and cystatin C levels in CKD patients were higher than in controls $(p<0.001$ vs. control group for all), while the hemoglobin level and eGFR in CKD patients were significantly lower than in controls $(p=0.002$ and $<0.001$, respectively) (Table 1$)$.

\section{Comparison of Kidney Volume and ERPF between the} Left and Right Kidneys in CKD Patients

There were no significant differences in the kidney volume or ERPF between the left and right kidneys in CKD patients ( $p=0.92$ and 0.46 , respectively) (Table 2 ).

\section{Comparison of Kidney R2* Values Obtained by 2}

BOLD-MRI Analysis Methods in the 2 Groups

The cortical, medullary, and whole-kidney R2* values in the CKD patients measured by the 2 BOLD-MRI analysis methods were significantly higher than those in the controls ( $p<0.01$ for each). The cortical, medullary, and whole-kidney R2* values of the left and right kidneys were not different in either the controls or the CKD patients 
( $p>0.05$ for each). The medullary and whole-kidney R2* values were higher than the cortical R2* values in the same group ( $p<0.001$ for each). Compared with the regional ROI selection method, the whole-kidney ROI selection method yielded higher cortical R2* values in both the controls and CKD patients ( $p<0.01$ for each) (Table 3$)$.

\section{Correlation between $R 2^{*}$ Values and Kidney Function}

Parameters in CKD Patients

The cortical and medullary R2* values measured by the regional ROI selection method had no correlations with age ( $p=0.35,0.30$, respectively), systolic blood pressure ( $p=0.67,0.80$, respectively), diastolic blood pressure ( $p=$ $0.75,0.43$, respectively), hemoglobin $(p=0.07,0.31$, respectively), Scr ( $p=0.13,0.38$, respectively), blood urea nitrogen $(p=0.09,0.23$, respectively), uric acid $(p=0.48,0.98$, respectively), cystatin $\mathrm{C}(p=0.20,0.32$, respectively), eGFR ( $p=0.78,0.34$, respectively), kidney volume ( $p=0.90,0.92$, respectively), or ERPF ( $p=0.54,0.18$, respectively) in the CKD patients. The cortical R2* values measured by the whole-kidney ROI selection method were not correlated with age ( $p=0.77)$, systolic blood pressure $(p=0.25)$, diastolic blood pressure $(p=0.88)$, hemoglobin $(p=0.96)$, Scr $(p=0.29)$, blood urea nitrogen $(p=0.28)$, uric acid $(p=$ $0.90)$, cystatin $\mathrm{C}(p=0.37)$, eGFR $(p=0.15)$, kidney volume $(p=0.12)$, or ERPF $(p=0.39)$ in the CKD patients. The whole-kidney R2* values measured by the whole-kidney ROI selection method were positively correlated with the $\operatorname{Scr}(r=0.312, p=0.05)$, blood urea nitrogen $(r=0.421, p=$ $0.007)$ and cystatin $\mathrm{C}(r=0.31, p=0.05)$ levels and inversely correlated with the eGFR $(r=-0.472, p=0.01)$ and ERPF $(r=-0.542, p=0.002)$. However, the whole-kidney R2* values showed no correlations with age $(p=0.99)$, systolic blood pressure $(p=0.52)$, diastolic blood pressure $(p=$ $0.15)$, hemoglobin $(p=0.59)$, uric acid $(p=0.44)$, or kidney volume ( $p=0.06$ ) in the CKD patients (Table 4; Fig. 3 ).

\section{Comparison of Kidney T2* Images in Controls and CKD Patients}

In the control group, the size and shape of the kidneys were normal, and the renal cortico-medullary zones were clear. However, compared with the controls, in the CKD patients, the size of the kidney was smaller, the shape was irregular, the renal cortex was thinner, and the renal cortico-medullary zones were unclear (Fig. 4A, B).

\section{Comparison of Kidney R2* Images in Controls and CKD Patients}

In the controls, the renal cortex was homogeneously blue, indicating abundant cortical oxygenation, and the
Table 2. Kidney volume and ERPF in CKD patients

\begin{tabular}{llll}
\hline Measurement & $\begin{array}{l}\text { Left } \\
\text { kidney }\end{array}$ & $\begin{array}{l}\text { Right } \\
\text { kidney }\end{array}$ & $\begin{array}{l}p \\
\text { value }\end{array}$ \\
\hline $\begin{array}{l}\text { MRI } \\
\quad \text { Kidney volume, } \mathrm{cm}^{3}(n=40)\end{array}$ & $219.4 \pm 87.4$ & $217.6 \pm 71.1$ & 0.92 \\
$\begin{array}{l}\text { ECT } \quad \text { ERPF, } \mathrm{mL} / \min (n=29) \\
\text { n }\end{array}$ & $84.8 \pm 49.1$ & $94.9 \pm 53.0$ & 0.46 \\
\hline
\end{tabular}

The data are expressed as the mean \pm standard deviation. MRI, magnetic resonance imaging; ECT, emission computed tomography; ERPF, effective renal plasma flow.

medulla of the kidney was green and yellow, indicating that the medullary oxygenation level was lower than the cortical oxygenation level. Although the renal cortex was also blue in the CKD patients, the color distribution was uneven, and green and yellow were locally distributed in the cortex. In addition, there were large green, yellow, and red areas in the medulla in the CKD patients, particularly in the deep medulla. These color changes in the R2* maps of the kidneys of the CKD patients show that, compared with the controls, the cortical and medullary oxygenation levels are decreased and the reduced oxygenation in the medulla is more pronounced in the CKD patients (Fig. 4C, D).

\section{Comparison of Kidney Function Parameters and}

Kidney R2* Values $\left(s^{-1}\right)$ Obtained by Whole-Kidney

ROI Selection Method in Stable Renal Function Group

and Deteriorating Renal Function Group after 6-12

Months

After 6-12 months, the Scr, uric acid, and eGFR levels in 2 groups were significantly different $(p=0.023,0.038$, and $<0.001$, respectively), while the hemoglobin, blood urea nitrogen, and cystatin C levels were not significantly different ( $p=0.665,0.169$, and 0.877 , respectively) (Table 5). All the kidney function parameters levels in 2 groups were not significantly different, while the wholekidney R2* values in deteriorating renal function group were significantly higher than those instable renal function group at baseline ( $p=0.033$ and 0.042 , respectively) (Table 6).

\section{Discussion}

Using 2 BOLD-MRI analysis methods to analyze renal oxygenation in healthy volunteers, we found that the medullary and whole-kidney R2* values were higher than 
Table 3. Kidney R2* values $\left(\mathrm{s}^{-1}\right)$ obtained by 2 BOLD-MRI analysis methods in controls and CKD patients

\begin{tabular}{|c|c|c|c|c|}
\hline \multirow[t]{2}{*}{ Measurement } & \multicolumn{2}{|c|}{ Controls $(n=10)$} & \multicolumn{2}{|c|}{ CKD patients $(n=40)$} \\
\hline & left kidney & right kidney & left kidney & right kidney \\
\hline \multicolumn{5}{|l|}{ Regional ROI selection } \\
\hline Cortical R2* & $17.2 \pm 1.4$ & $17.2 \pm 1.2$ & $20.2 \pm 3.0^{*}$ & $20.2 \pm 3.0^{*}$ \\
\hline Medullary R2* & $23.6 \pm 0.9^{\#}$ & $23.8 \pm 1.1^{\#}$ & $31.8 \pm 3.2^{*, \#}$ & $31.8 \pm 3.5^{*, \#}$ \\
\hline \multicolumn{5}{|c|}{ Whole-kidney ROI selection } \\
\hline Cortical R2* & $21.8 \pm 0.8^{\#}$ & $22.2 \pm 1.1^{\#}$ & $25.9 \pm 3.5^{*, \#}$ & $25.7 \pm 3.2^{*, \#}$ \\
\hline Whole-kidney R2* & $26.2 \pm 1.2^{\&}$ & $27.1 \pm 1.4^{\&}$ & $34.3 \pm 3.4^{*, \&}$ & $34.2 \pm 3.4^{*, \&}$ \\
\hline $\begin{array}{l}\text { The data are expres } \\
* p<0.01 \text { versus the it } \\
\text { values in the same grov } \\
\text { same group using who }\end{array}$ & $\begin{array}{l}\text { standard de } \\
\text { R2* values } \\
\text { 1 ROI select } \\
\text { election. }\end{array}$ & $\begin{array}{l}\text { CKD, chroni } \\
\text { ontrols. }{ }^{\#} p< \\
0.001 \text { versus }\end{array}$ & $\begin{array}{l}\text { ey disease; R } \\
\text { versus the ip } \\
\text { osilateral cort }\end{array}$ & $\begin{array}{l}\text { on of interest. } \\
\text { l cortical R2* } \\
\text { * values in the }\end{array}$ \\
\hline
\end{tabular}

the cortical R2* values. Li et al. [21] also found that the renal medulla R2* values were significantly higher than that of cortex in the healthy. It indicates that the cortical oxygenation level was high, while the medullary oxygenation level was relatively low in the physiological state, and the blood distribution of them was different. In our study, the cortical, medullary, and whole-kidney oxygenation levels were significantly decreased in the CKD patients compared with the healthy volunteers. We also found that medullary oxygenation was higher than cortical oxygenation using the regional ROI selection method. The reasons for these results are related not only to the low perfusion and high oxygen consumption in the medulla but also to the imaging characteristics of BOLDMRI. In renal tissue, the oxygen dissociation curve is flat when the partial oxygen pressure is $>60 \mathrm{~mm} \mathrm{Hg}$, while the oxygen dissociation curve is steeper when the partial oxygen pressure is $<26.6 \mathrm{~mm} \mathrm{Hg}$ [22]. Typically, the partial pressure of oxygen in most cortical regions is $>60 \mathrm{~mm} \mathrm{Hg}$, while the partial pressure of oxygen in medullary zones is $<26.6 \mathrm{~mm} \mathrm{Hg}$. Therefore, even if the partial oxygen pressure changes slightly in the medulla, this change will lead to a significant increase in the content of deoxyhemoglobin. These circumstances enable the sensitivity and accuracy of evaluating medullary oxygenation via BOLD-MRI based on deoxyhemoglobin.

In addition, we found that compared with the regional ROI selection method, the whole-kidney ROI selection method yielded higher cortical R2* values in the healthy volunteers and the CKD patients. The reasons for this result are related to the renal physiological characteristics of CKD patients and the BOLD-MRI analysis methods. First, in CKD, the kidney volume is significantly reduced, and the cortico-medullary zones are unclear. ROIs in the cortex easily include cortico-medullary overlap zones. The ROI placed in the whole-kidney ROI selection method contains more cortical and cortico-medullary zones, resulting in a higher cortical R2* value. Second, in CKD, the areas of hypoxia in the cortex are scattered. This means that if the 3 ROIs selected in the regional ROI selection method do not contain hypoxic regions, the cortical R2* value obtained by this method will be larger than that obtained by the whole-kidney ROI selection method, in which most of the cortical area is included in the ROI. Similarly, the medullary R2* value measured by the regional ROI selection method has high variability due to observer bias. To overcome these shortcomings, in the whole-kidney ROI selection method, the medullary R2* value is not evaluated. Instead, 1 ROI is used to trace the entire kidney region (excluding the collection system, cysts, and hilar vessels) to measure the oxygenation of the whole kidney.

In our study, there were no significant differences in the kidney R2* values, kidney volume, or ERPF between the left and right kidneys in CKD patients. Therefore, the parameters of the right kidney in CKD patients were selected to analyze the correlations between the kidney R2* values and kidney function and ERPF in this experiment. We found that the cortical, medullary, and whole-kidney $\mathrm{R} 2 *$ values were not related to age, blood pressure, hemoglobin, uric acid, or kidney volume in the CKD patients, which is in agreement with some results obtained by other researchers. Michaely et al. [23] found that the cortical and medullary R2* values were not affected by sex or age in CKD patients using the regional ROI selection method. Pruijm et al. [24] also found that the cortical and medul- 
Table 4. Relationships of R2* values $\left(\mathrm{s}^{-1}\right)$ with age, SBP, DBP, hemoglobin, Scr, BUN, uric acid, cystatin C, eGFR, kidney volume, and ERPF in CKD patients

\begin{tabular}{|c|c|c|c|c|}
\hline \multirow[t]{2}{*}{ Measurement } & \multicolumn{2}{|c|}{ Regional ROI selection } & \multicolumn{2}{|c|}{ Whole-kidney ROI selection } \\
\hline & cortical R2* & medullary R2* & cortical R2* & whole kidney R2* \\
\hline \multicolumn{5}{|l|}{ Age } \\
\hline$r$ & 0.150 & 0.169 & 0.048 & 0.003 \\
\hline \multirow{2}{*}{\multicolumn{5}{|c|}{ SBP/DBP }} \\
\hline & & & & \\
\hline$r$ & $-0.070 /-0.052$ & $0.041 / 0.128$ & $-0.187 / 0.025$ & $0.104 / 0.230$ \\
\hline$p$ & $0.67 / 0.75$ & $0.80 / 0.43$ & $0.25 / 0.88$ & $0.52 / 0.15$ \\
\hline \multicolumn{5}{|l|}{ Hemoglobin } \\
\hline$r$ & -0.290 & -0.165 & -0.009 & -0.088 \\
\hline$p$ & 0.07 & 0.31 & 0.96 & 0.59 \\
\hline \multicolumn{5}{|l|}{ Scr } \\
\hline$r$ & 0.244 & 0.142 & 0.173 & 0.312 \\
\hline \multirow{2}{*}{\multicolumn{5}{|c|}{$\begin{array}{c}p \\
\text { BUN }\end{array}$}} \\
\hline & & & & \\
\hline$r$ & 0.274 & 0.200 & 0.175 & 0.421 \\
\hline$p$ & 0.09 & 0.22 & 0.28 & 0.007 \\
\hline \multicolumn{5}{|l|}{ Uric acid } \\
\hline$r$ & 0.115 & -0.005 & -0.022 & 0.125 \\
\hline$p$ & 0.48 & 0.98 & 0.89 & 0.44 \\
\hline \multicolumn{5}{|l|}{ Cystatin C } \\
\hline$r$ & 0.208 & 0.160 & 0.145 & 0.310 \\
\hline \multirow{2}{*}{\multicolumn{5}{|c|}{ eGFR }} \\
\hline & & & & \\
\hline$r$ & -0.055 & -0.184 & -0.276 & -0.472 \\
\hline$p$ & 0.78 & 0.34 & 0.15 & 0.01 \\
\hline \multicolumn{5}{|l|}{ Kidney volume } \\
\hline$r$ & -0.024 & 0.018 & -0.287 & -0.332 \\
\hline$p$ & 0.90 & 0.92 & 0.11 & 0.06 \\
\hline \multicolumn{5}{|l|}{ ERPF } \\
\hline$r$ & 0.120 & -0.255 & -0.166 & -0.542 \\
\hline$p$ & 0.54 & 0.18 & 0.39 & 0.002 \\
\hline
\end{tabular}

SBP, systolic blood pressure; DBP, diastolic blood pressure; Scr, serum creatinine; BUN, blood urea nitrogen; eGFR, estimated glomerular filtration rate; ERPF, effective renal plasma flow; ROI, region of interest. The wholekidney R $2 *$ values measured by the whole-kidney ROI selection method were positively correlated with the serum creatinine $(r=0.312, p=0.05)$, blood urea nitrogen $(r=0.421, p=0.007)$ and cystatin $\mathrm{C}(r=0.31, p=0.05)$ levels and inversely correlated with the eGFR $(r=-0.472, p=0.01)$ and ERPF $(r=-0.542, p=0.002)$.

lary R2* values measured using the regional ROI selection method in CKD patients were not related to age or blood pressure. However, it is interesting that the cortical R2* value was positively correlated with the level of uric acid because hyperuricemia can constrict cortical blood vessels, thereby reducing cortical oxygenation. Prasad et al. [25] found that the hemoglobin and cortical R2* values in CKD patients were both higher than those in anemic patients, indicating that hemoglobin had little effect on cortical oxygenation. Currently, there are few reports on the relationship between the kidney volume and renal tissue oxygenation in CKD patients. Our study found no sig- nificant relationship between the whole-kidney R2* value and kidney volume in CKD patients, but the correlation coefficient was high, which merits further study.

The whole-kidney R2* value measured using the wholekidney ROI selection method was positively correlated with the Scr, blood urea nitrogen, and cystatin C levels and negatively correlated with the eGFR and ERPF in CKD patients in this experiment. These results suggest that a reduction in whole-kidney oxygenation is closely related to reductions in kidney function and ERPF in CKD. Saad et al. [19] found that the decrease in renal parenchyma oxygenation measured by fractional tissue hypoxia, compared with that mea- 


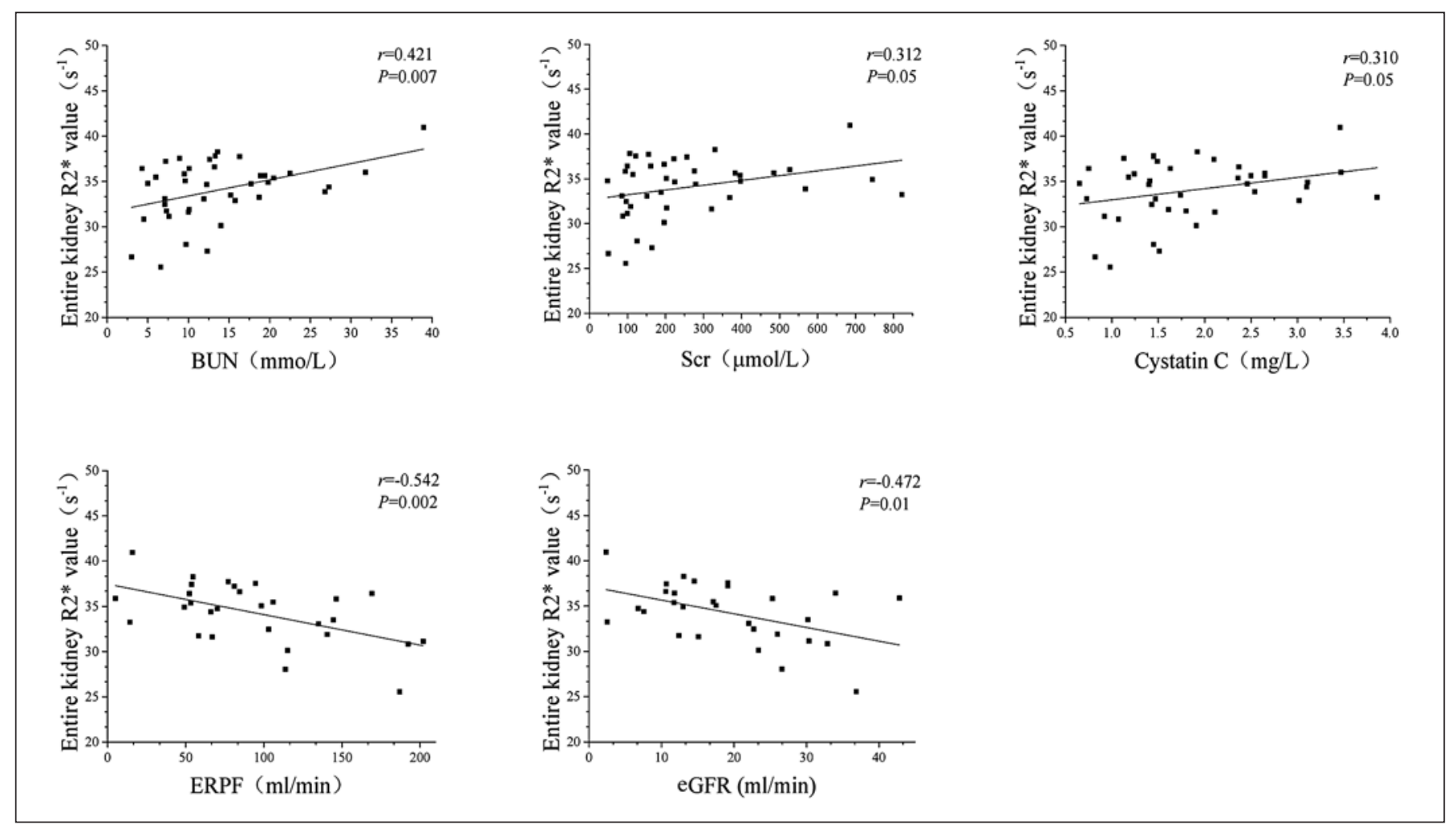

Fig. 3. The whole-kidney R2* values measured by the whole-kidney ROI selection method were positively correlated with the Scr, blood urea nitrogen, and cystatin C levels and inversely correlated with the eGFR and ERPF. eGFR, estimated glomerular filtration rate; ROI, region of interest; ERPF, effective renal plasma flow; Scr, serum creatinine.

sured by the regional ROI selection method, was closely related to decreases in the renal blood flow, tissue perfusion, and GFR in atherosclerotic renal artery stenosis patients; diuretics could improve the medullary oxygenation level. Warner et al. [20] and Pruijm et al. [26] have also shown that diuretics can rapidly reduce medullary oxygen consumption by blocking sodium transport in the ascending thick limb of Henle's loop. However, clinical researchers usually pay more attention to decreases in kidney function and ignore the effects of renal tubule dysfunction on the progression of kidney disease.

Recent studies have reported that the evaluation of oxygenation in renal cortex by BOLD-MRI can predict the progression of renal function [17, 27]. Our experiment also found the whole-kidney R2* values obtained by whole-kidney ROI selection method can predict the progression of CKD earlier than the hematology indicators. However, Michaely et al. [23] found no correlation between the cortical and medullary R2* values and the GFR in CKD patients at different stages. Pruijm et al. [24] found no differences in the cortical or medullary R2* val- ue measured by the regional ROI selection method among healthy volunteers, participants with hypertension and CKD patients. This may be related to regional ROI selection method's subjectivity and limitations. Therefore, the whole-kidney oxygenation level measured using the whole-kidney ROI selection method is a more accurate and reliable evaluation and may have an early prediction of the progression of CKD.

\section{Conclusions}

Compared with the healthy controls, the CKD patients exhibited significantly decreased renal oxygenation in the cortex and the medulla, particularly in the medulla. The decrease in whole-kidney oxygenation was closely related to decrease in kidney function, renal glomerular filtration, and tubular function. The whole-kidney R2* value measured using the whole-kidney ROI selection method may be a superior reflection and predictor of the progression of CKD. 


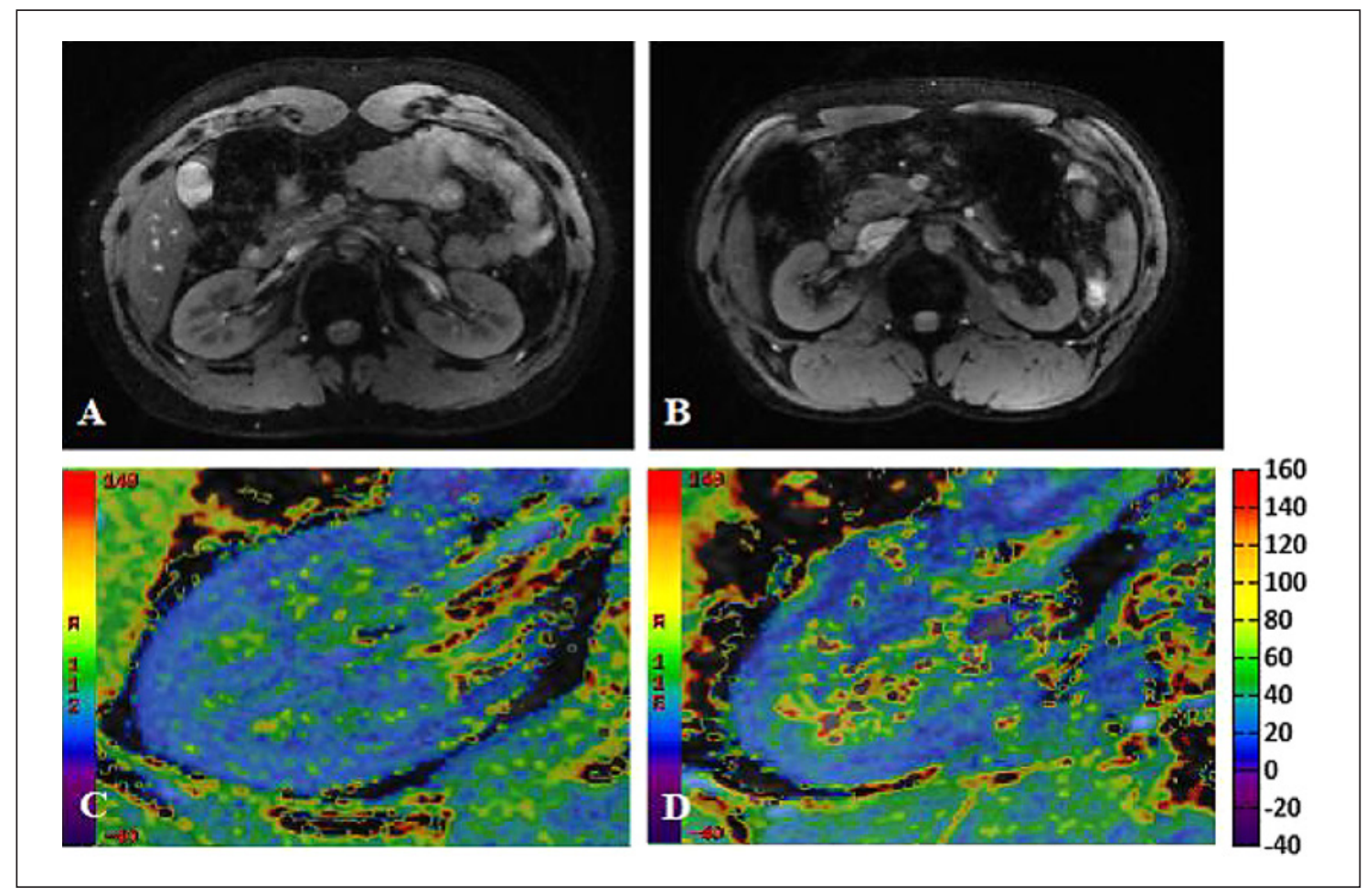

Fig. 4. T2* maps: A Controls: the size and shape of the kidney were normal, and the renal cortico-medullary zones were clear. B CKD patients: the size of the kidney was small, the shape of the kidney was irregular, and the renal cortico-medullary zones were unclear. R2* maps: C Controls: homogeneous blue in the cortex indicates abundant cortical oxygenation. Green and yellow in the medulla indicate decreased medullary oxygenation. D CKD patients: uneven blue in the cortex indicates decreased cortical oxygenation. The large green, yellow, and red areas in the medulla indicate decreased medullary oxygenation, which is more pronounced in the deep medulla.

Table 5. Comparison of kidney function parameters in stable renal function group and deteriorating renal function group after 6-12 months

\begin{tabular}{lccc}
\hline Parameters & $\begin{array}{l}\text { Stable renal function } \\
\text { group }(n=15)\end{array}$ & $\begin{array}{l}\text { Deteriorating renal } \\
\text { function group }(n=14)\end{array}$ & $p$ value \\
\hline Hemoglobin, g/L & $113.60 \pm 27.18$ & $95.14 \pm 40.77$ & 0.665 \\
Scr, $\mu$ mol/L & $192.27 \pm 178.38$ & $459.29 \pm 358.30$ & $\mathbf{0 . 0 2 3}$ \\
Blood urea nitrogen, $\mathrm{mmol} / \mathrm{L}$ & $11.41 \pm 8.14$ & $20.01 \pm 14.23$ & 0.169 \\
Uric acid, $\mu \mathrm{mol} / \mathrm{L}$ & $388.53 \pm 138.17$ & $412.07 \pm 131.83$ & $\mathbf{0 . 0 3 8}$ \\
Cystatin C, $\mathrm{mg} / \mathrm{L}$ & $1.67 \pm 1.10$ & $2.71 \pm 1.31$ & 0.877 \\
eGFR, $\mathrm{mL} / \mathrm{min}$ & $64.34 \pm 44.28$ & $24.96 \pm 17.85$ & $<\mathbf{0 . 0 0 1}$ \\
\hline
\end{tabular}

The data are expressed as the mean \pm standard deviation or as a numerical value. eGFR, estimated glomerular filtration rate; Scr, serum creatinine. The serum creatinine, uric acid and eGFR levels in two groups were significantly different $(p=0.023,0.038$ and $<0.001$, respectively).

\section{Limitations}

Our study failed to analyze the differences in the renal oxygenation levels of CKD patients at different stages because of the few available samples. Although the shape of the renal cortex and the whole-kidney delineated by the whole-kidney ROI selection method was the same as that determined by fractional kidney hypoxia, the cortical and whole-kidney R2* values were quantitative in this study. However, whether quantitative whole-kidney R2* values can demonstrate changes in kidney function better than 
Table 6. Comparison of kidney function parameters and kidney R2* values $\left(\mathrm{s}^{-1}\right)$ obtained by whole-kidney ROI selection method in stable renal function group and deteriorating renal function group at baseline

\begin{tabular}{lccc}
\hline Parameters & $\begin{array}{l}\text { Stable renal function } \\
\text { group }(n=15)\end{array}$ & $\begin{array}{l}\text { Deteriorating renal } \\
\text { function group }(n=14)\end{array}$ & $p$ value \\
\hline Hemoglobin, g/L & $117.93 \pm 29.53$ & $101.14 \pm 41.50$ & 0.439 \\
Scr, $\mu$ mol/L & $248.27 \pm 244.02$ & $268.21 \pm 185.23$ & 0.805 \\
Blood urea nitrogen, mmol/L & $10.96 \pm 5.78$ & $15.72 \pm 9.98$ & 0.135 \\
Uric acid, $\mu \mathrm{mol} / \mathrm{L}$ & $451.27 \pm 161.45$ & $417.57 \pm 119.83$ & 0.315 \\
Cystatin C, mg/L & $1.68 \pm 1.01$ & $2.01 \pm 0.74$ & 0.527 \\
eGFR, mL/min & $55.76 \pm 43.53$ & $33.20 \pm 23.84$ & 0.095 \\
Right whole-kidney R2*, $\mathrm{s}^{-1}$ & $32.1 \pm 3.7$ & $34.9 \pm 3.0$ & $\mathbf{0 . 0 3 3}$ \\
Left whole-kidney R2*, $\mathrm{s}^{-1}$ & $32.6 \pm 3.4$ & $35.3 \pm 3.4$ & $\mathbf{0 . 0 4 2}$ \\
\hline
\end{tabular}

The data are expressed as the mean \pm standard deviation or as a numerical value. eGFR, estimated glomerular filtration rate; ROI, region of interest; Scr, serum creatinine. The whole-kidney R2* values in deteriorating renal function group were significantly higher than those instable renal function group at baseline $(p=0.033$ and 0.042 , respectively).

fractional kidney hypoxia remains to be further studied. In addition, due to the limitation of experimental conditions, creatinine of each participant was only measured twice which may limit the appreciation of the yearly eGFR decline.

\section{Acknowledgements}

We gratefully acknowledge the work of past and present members of our research group and hospital.

\section{Statement of Ethics}

The research was conducted in accordance with the World Medical Association Declaration of Helsinki and complies with the guidelines for human studies. Written informed consent was obtained from all patients, and the Ethics Committee of Affiliated Hospital of Xuzhou Medical University approved this study (XYFY2016-KL031-01).

\section{Conflict of Interest Statement}

The authors declare that there are no conflicts of interest.

\section{Funding Sources}

This study was supported by funding from the National Natural Science Foundation of China (81270769) and Jiangsu Provincial Post Graduate Innovation Plan (KYCX17_1708, SJCX17_0560, KYCX18-2178, SJCX18_0715).

\section{Author Contributions}

Methodology and writing-original draft preparation, Fen Chen; formal analysis and writing-review and editing, Han Yan and Fan Yang; performing BOLD-MRI, Li Cheng and Siwei Zhang; data curation, Shulin Li and Caixia Liu; supervision and project administration, Kai Xu and Dong Sun.

\section{References}

1 Neugarten J. Renal BOLD-MRI and assessment for renal hypoxia. Kidney Int. 2012; 81(7):613-4.

2 Hirakawa Y, Tanaka T, Nangaku M. Renal hypoxia in CKD; pathophysiology and detecting methods. Front Physiol. 2017;8:99.

3 Nangaku M. Hypoxia and tubulointerstitial injury: a final common pathway to end-stage renal failure. Nephron Exp Nephrol. 2004; 98(1):e8-12.

4 Palm F, Nordquist L. Renal tubulointerstitial hypoxia: cause and consequence of kidney dysfunction. Clin Exp Pharmacol Physiol. 2011;38(7):474-80

5 Ebrahimi B, Textor SC, Lerman LO. Renal relevant radiology: renal functional magnetic resonance imaging. Clin J Am Soc Nephrol. 2014;9(2):395-405.

6 Niles DJ, Gordon JW, Fain SB. Effect of anesthesia on renal R2* measured by blood oxygen level-dependent MRI. NMR Biomed. 2015;28(7):811-7.

7 Gloviczki ML, Glockner JF, Crane JA, McKusick MA, Misra S, Grande JP, et al. Blood oxy- gen level-dependent magnetic resonance imaging identifies cortical hypoxia in severe renovascular disease. Hypertension. 2011; 58(6):1066-72.

8 Gloviczki ML, Lerman LO, Textor SC. Blood oxygen level-dependent (BOLD) MRI in renovascular hypertension. Curr Hypertens Rep. 2011;13(5):370-7.

9 Yin WJ, Liu F, Li XM, Yang L, Zhao S, Huang $\mathrm{ZX}$, et al. Noninvasive evaluation of renal oxygenation in diabetic nephropathy by BOLDMRI. Eur J Radiol. 2012;81(7):1426-31. 
10 Xin-Long P, Jing-Xia X, Jian-Yu L, Song W, Xin-Kui T. A preliminary study of blood-oxygen-level-dependent MRI in patients with chronic kidney disease. Magn Reson Imaging. 2012;30(3):330-5.

11 Bauer F, Wald J, Bauer FJ, Dahlkamp LM, Seibert FS, Pagonas N, et al. Detection of acute tubular necrosis using blood oxygenation level-dependent (BOLD) MRI. Kidney Blood Press Res. 2017;42(6):1078-89.

12 Park SY, Kim CK, Park BK, Kim SJ, Lee S, Huh W. Assessment of early renal allograft dysfunction with blood oxygenation level-dependent MRI and diffusion-weighted imaging. Eur J Radiol. 2014;83(12):2114-21.

13 Khatir DS, Pedersen M, Jespersen B, Buus $\mathrm{NH}$. Evaluation of renal blood flow and oxygenation in CKD using magnetic resonance imaging. Am J Kidney Dis. 2015;66(3):40211.

14 Ebrahimi B, Gloviczki M, Woollard JR, Crane JA, Textor SC, Lerman LO. Compartmental analysis of renal BOLD MRI data: introduction and validation. Invest Radiol. 2012;47(3): $175-82$.

15 Gloviczki ML, Saad A, Textor SC. Blood oxygen level-dependent (BOLD) MRI analysis in atherosclerotic renal artery stenosis. Curr Opin Nephrol Hypertens. 2013;22(5):519-24.
16 Piskunowicz M, Hofmann L, Zuercher E, Bassi I, Milani B, Stuber M, et al. A new technique with high reproducibility to estimate renal oxygenation using BOLD-MRI in chronic kidney disease. Magn Reson Imaging. 2015; 33(3):253-61.

17 Pruijm M, Milani B, Pivin E, Podhajska A, Vogt B, Stuber M, et al. Reduced cortical oxygenation predicts a progressive decline of renal function in patients with chronic kidney disease. Kidney Int. 2018;93(4):932-40.

18 Li LP, Milani B, Pruijm M, Kohn O, Sprague S, Hack B, et al. Renal BOLD MRI in patients with chronic kidney disease: comparison of the semi-automated twelve layer concentric objects (TLCO) and manual ROI methods. MAGMA. 2020;33(1):113-20.

19 Saad A, Crane J, Glockner JF, Herrmann SM, Friedman H, Ebrahimi B, et al. Human renovascular disease: estimating fractional tissue hypoxia to analyze blood oxygen level-dependent MR. Radiology. 2013;268(3):770-8.

20 Warner L, Glockner JF, Woollard J, Textor SC, Romero JC, Lerman LO. Determinations of renal cortical and medullary oxygenation using blood oxygen level-dependent magnetic resonance imaging and selective diuretics. Invest Radiol. 2011;46(1):41-7.

21 Li C, Liu H, Li X, Zhou L, Wang R, Zhang Y. Application of BOLD-MRI in the classification of renal function in chronic kidney disease. Abdom Radiol. 2019;44(2):604-11.
22 Djamali A, Sadowski EA, Samaniego-Picota M, Fain SB, Muehrer RJ, Alford SK, et al. Noninvasive assessment of early kidney allograft dysfunction by blood oxygen level-dependent magnetic resonance imaging. Transplantation. 2006;82(5):621-8.

23 Michaely HJ, Metzger L, Haneder S, Hansmann J, Schoenberg SO, Attenberger UI. Renal BOLD-MRI does not reflect renal function in chronic kidney disease. Kidney Int. 2012;81(7):684-9.

24 Pruijm M, Hofmann L, Piskunowicz M, Muller ME, Zweiacker C, Bassi I, et al. Determinants of renal tissue oxygenation as measured with BOLD-MRI in chronic kidney disease and hypertension in humans. PLoS One. 2014;9(4):e95895.

25 Prasad PV, Thacker J, Li LP, Haque M, Li W, Koenigs $\mathrm{H}$, et al. Multi-parametric evaluation of chronic kidney disease by MRI: a Preliminary Cross-Sectional Study. PLoS One. 2015; 10(10):e0139661.

26 Pruijm M, Hofmann L, Maillard M, Tremblay S, Glatz N, Wuerzner G, et al. Effect of sodium loading/depletion on renal oxygenation in young normotensive and hypertensive men. Hypertension. 2010;55(5):1116-22.

27 Zhou H, Yang M, Jiang Z, Ding J, Di J, Cui L. Renal hypoxia: an important prognostic marker in patients with chronic kidney disease. Am J Nephrol. 2018;48(1):46-55. 